\title{
Comparative study on the independent and combined effects of omega- 3 and vitamin B12 on phospholipids and phospholipase A2 as phospholipid hydrolyzing enzymes in PPA-treated rats as a model for autistic traits
}

Hanan Alfawaz ${ }^{1}$, Ramesa Shafi Bhat ${ }^{2}$, Manar Al-Mutairi ${ }^{3}$, Osima M. Alnakhli ${ }^{3}$, Abeer Al-Dbass ${ }^{2}$, Mona AlOnazi ${ }^{2}$, Majidh Al-Mrshoud ${ }^{3}$ Iman H. Hasan ${ }^{4}$ and Afaf El-Ansary ${ }^{3^{*}}$ (D)

\begin{abstract}
Background: Abnormal phospholipid metabolism is a major component of many neurodevelopmental disorders including autism. Oral administration of propionic acid (PPA) can produce behavioral abnormalities and biochemical features in rodents similar to those observed in autism and can thus be used as a model to understand impaired brain fatty acid metabolism in autism.

Methods: The present study was designed to understand alterations in phospholipid metabolism in the brain of a rodent model of autism and to explore omega- 3 and vitamin B12 as remedies. Five groups of rats were selected: Group 1 was the control. Group 2 was the rodent model of autism treated with a neurotoxic dose of PPA. Group 3 was given vitamin B12 cobalamin (16.7 mg/kg/day) for 30 days after PPA treatment. Group 4 was given pharmaceutical grade Omega-3 (200 mg cholesterol free-DHA/kg body weight/day), a product of Madre lab, Germany, for 30 days after PPA treatment for 3 days. Group 5 was given a combined dose of $\omega-3+$ Vitamin B12 for the same duration postPPA treatment. Phospholipid levels and Phospholipase A2 were measured in the brain homogenates of all the groups. ELISA and western blotting were used to detect the CPLA2 protein level.
\end{abstract}

Results: A significant decrease in phospholipid levels and a significant increase in CPLA2 were found in brain tissue of PPA-treated rats; however, both $\omega-3$ and vitamin B12 were efficient in ameliorating the neurotoxic effect of PPA.

Conclusion: Both $\omega-3$ and vitamin B12 may play a role in ameliorating impaired phospholipid metabolism in autism; however, proper clinical trials are needed.

Keywords: Autism, Propionic acid, Omega-3, Vitamin B12, Phospholipase A2, Phospholipids

\footnotetext{
* Correspondence: afafkelansary@gmail.com

${ }^{3}$ Central Laboratory, Center for Female Scientific and Medical Colleges, King

Saud University, Riyadh, Saudi Arabia

Full list of author information is available at the end of the article
}

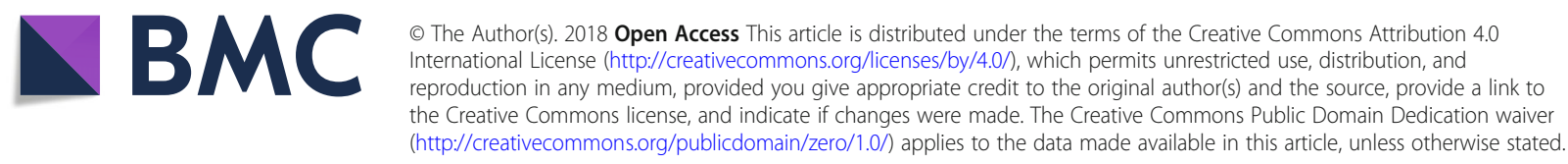




\section{Background}

Propionic acid (PPA) is a short-chain fatty acid produced in the gut as a metabolite of Clostridium difficile species and is higher in feces of autistic patients than of normal controls. El-Ansary et al. [1] designed a rodent model of autism by inducing persistent biochemical autistic features by oral administration of PPA in rat pups, including oxidative stress, neuro-inflammation, altered neurochemistry, and impaired energy and lipid metabolism. Recently, the same model with identical dose and mode of administration of PPA was effective in inducing several behavioral abnormalities, such as social interaction impairment, hyperactivity, and repetitive behaviors, together with histopathological changes such as neuronal loss and astrogliosis $[2,3]$. This finding supports the use of PPA in animal models of autism.

Total phospholipids account for $20-25 \%$ of brain dry weight and form the major components of bio-membranes. Total phospholipids are critical for brain fluidity and ion permeability as dynamic factors that clearly affect cognition [4]. Dysregulated metabolism characterized by lower levels of plasma and brain phospholipids has been proposed as an etiological mechanism in autism [5]. Moreover, phospholipid fatty acid profiles are thought to be an accurate biomarker for the fatty acid status of the brain [4].

Phospholipid components, such as phosphatidylcholine (PC), phosphatidylethanolamine (PE), phosphatidylethanolamine plasmalogen (PEpl), phosphatidylserine (PS) and phosphoinositides (PI, PIP and PIP2), of the mammalian brain are very important for normal brain development and function. In addition to their function as integral components of membrane structure, PS and PI have unique functions. PI and its phosphorylated derivatives are linked to G-protein coupled phospholipase C (PLC) for the release of inositol trisphosphates (IP3) and diacylglycerol (DG), which in turn serve as second messengers for mobilization of intracellular calcium stores and activation of protein kinase $\mathrm{C}$ (PKC), respectively [6-10].

Interestingly, during the third trimester of pregnancy and the first two years of life, the brain undergoes a period of rapid development that is critically affected by nutrient insufficiency. DHA is a nutrient that is required for the proper development of the cognitive, sensory, motor, and perceptual systems during this period [11, 12]. Based on the fact that the neurons are continually forming axons and dendritic extensions with accompanying cell membranes, the growing membrane must be relatively fluid; thus, DHA, as the element producing the most fluidity in cell membranes, is highly recommended as a supplement during this neurodevelopment period. Additionally, the synapses, as the primary functional units of brain circuits, are preferentially made from membranes with adequate DHA [13].

Omega-3 and omega-6 PUFA metabolism occurs through the same pathway as desaturase and elongase enzyme activity. Through this pathway, the essential substrate PUFA n-6 linoleic (LA) and n-3 $\alpha$-linolenic (ALA) are transformed into n-6 AA and n-3 EPA/DHA, respectively. Through the cyclooxygenase (COX) and lipoxygenase (LOX) pathways, AA and EPA are converted into eicosanoids and lipid mediators. Since PUFA are critically important in brain maturation, rapid conversion of these fatty acids to their metabolites may be implicated in the phenotypic brain abnormalities existing in individuals with autism.

Arachidonic acid (AA) and a number of AA-cascade lipid mediators, presented by prostaglandins (PGs), leukotrienes and other eicosanoids, are well documented to exert a variety of neuromodulatory actions such as wakefulness/sleep regulation, pain sensation, and neuroendocrine regulation $[14,15]$. The initial step of the AA $(\omega-6)$ signaling cascade is regulated by the action of phospholipase A2 (PLA2) which catalyzes the breakdown of membrane phospholipids, releasing AA. There are three known types of PLA2, $\mathrm{Ca}^{2+}$-sensitive, cytosolic PLA2 (cPLA2), the secreted form of PLA2 (sPLA2), and $\mathrm{Ca}^{2+}$-insensitive enzyme (iPLA2). Cytosolic PLA2 showed high specificity to AA and catalyzes the release of AA from the $s n-2$ position of phospholipids [16]. Cytosolic PLA2 has been shown to play a critical role in neuronal plasticity and glutamate excitotoxicity [17].

Saluja et al. [18] reported that both CPLA2 activity and protein levels are significantly increased during brain ischemia. In astrocyte-neuron cocultures, chronic glutamate toxicity was found to induce the activation of astrocytic PLA2. This process was usually followed by significant increase of AA into the medium and increase of lipoxygenase (LOX) and cyclooxygenase (COX) activities, leading to neuronal death [19]. Interestingly, cPLA2 $\alpha$ in mice have lower levels of brain AA, COX-2 mRNA, and protein levels. This finding may suggest that cPLA2 and COX-2 are functionally coupled and that overexpression of these enzymes can be easily related to brain neuroinflammation, previously reported in brain homogenates of rats or hamsters after oral administration of a neurotoxic dose of PPA $[1,20,21]$. Pharmacological studies demonstrate that inhibition of cPLA2 may be promising for the treatment of neuro-inflammation, the etiological mechanism involved in many neurological disorders including autism [22].

Our most recent work showed glutamate excitotoxicity and the impairment of the glutamine-glutamate-GABA circuit as two etiological mechanisms in PPA-induced neurotoxicity [23]. This result motivated our interest in measuring PLA2 protein concentration and phospholipids in brain homogenates of PPA-intoxicated rats and in determining the therapeutic effects of omega- 3 and vitamin B12 in ameliorating the neurotoxic effect of PPA.

Currently, western blotting is among the best techniques used to follow the effectiveness of treatments on specific target proteins as it greatly reduces cross-reactions which are a potential drawback of the ELISA technique [24]. In 
this work, the neurotoxic effects of PPA and the therapeutic effects of B12, $\omega-3$ and the mixture of both on PLA2 phospholipid hydrolyzing enzymes in a rodent model of autism were investigated. In addition, the sensitive and quantitative technique ELISA was used. Additionally, the presence of PLA2 was detected by western blotting.

\section{Methods}

- Animals: A total of 35 young male Western albino rats (80-120 g) were obtained from King Saud University, Riyadh. Rats were randomly allocated to the following groups with seven rats each: Group 1 was given only phosphate buffered saline and considered the control. Group 2 was the oral buffered PPA-treated group treated with the neurotoxic dose of $250 \mathrm{mg} / \mathrm{kg}$ body weight/day for 3 days [25]. Group 3 was given oral vitamin B12 with the dose of $16.7 \mathrm{mg}$ cobalamin/ $\mathrm{kg}$ body weight/ day, a product of Dawa Pharmaceutical Co., Limited, Hebei, and China, for 30 days after PPA treatment. Group 4 was given 200 mg cholesterol free-DHA/kg body weight/day, a pharmaceutical grade product of Madre lab, Germany, after PPA treatment for 3 days. Group 5 was given a combined dose of $\omega-3+$ vitamin B12, for the same duration post-PPA treatment. All groups were kept at a controlled temperature (21 $\pm 1{ }^{\circ} \mathrm{C}$ ) with ad libitum access to food and water. All experiments were performed in accordance with national animal care guidelines and were preapproved by the faculty ethics committee, King Saud University.

\section{Sample collection}

At the end of the feeding trials, the animals were killed by decapitation; the brains isolated from sacrificed animals were washed, dissected into small pieces and homogenized in distilled water using a Teflon homogenizer. The homogenate was centrifuged at $650 \mathrm{~g}$ for $20 \mathrm{~min}$ to remove debris and kept at $-80^{\circ} \mathrm{C}$ until further use.

\section{Measurement of phospholipids}

Phospholipid levels were quantified using a phospholipid assay kit from Abnova (Abnova [Taiwan] Corporation) with a linear detection range for the colorimetric assay of $3-200 \mu \mathrm{M}$ and for the fluorimetric assay of $0.6-20 \mu \mathrm{M}$ phospholipid.

Measurement of CPLA2 protein using the ELISA technique Phospholipase A2 was measured using a kit based on the sandwich ELISA principle, a product of LSBio (Lifespan BioScience, Inc., North America) with a detection range of $3.12-200 \mathrm{ng} / \mathrm{ml}$.

\section{Measurement of CPLA2 protein using western blotting}

To investigate the effect of $\mathrm{B} 12$ and $\omega-3$ on $\mathrm{CPLA}_{2}$ expression in the brain, we performed western blotting as we recently reported. Briefly, brain samples were homogenized in RIPA buffer containing proteinase inhibitors. Total protein content was assayed using Bradford reagent, and $60 \mu \mathrm{g}$ protein was separated on SDS-PAGE, electro-transferred onto nitrocellulose membranes, and blocked in 5\% skimmed milk in TBS Tween 20. The blocked membranes were probed with rabbit anti-cPLA 2 and mouse anti-GAPDH primary antibodies (Santa Cruz Biotechnology-454 and Abcam 9482, respectively). After washing, the membranes were incubated with the secondary antibodies and developed using an enhanced chemiluminescence kit (Bio-Rad, USA). The blots were scanned and intensity of the obtained bands was quantified using ImageJ (NIH, USA). The results were normalized to GAPDH and are presented as percent of control.

\section{Statistical analysis}

The results of the present study were expressed as the means \pm S.D. All statistical comparisons between the control group and the four studied groups were performed using one-way analysis of variance (ANOVA) tests with Dunnett's test for multiple comparisons using SPSS (Chicago, IL, USA). Significance was assigned at the level of $p$ $<0.05$. Receiver operating characteristics (ROC) curve analysis was also performed. The area under the curve (AUC), the degrees of sensitivity and specificity, and cutoff values were calculated.

\section{Results}

Table 1 and Fig. 1 demonstrate the mean \pm S.D. and the percentage change of CPLA2 and phospholipids in the five studied groups. It is clear that PPA induced significant elevation in cPLA2 at 28.54\%, together with a significant decrease in phospholipids at $27.14 \%$. Both $\omega-3$ and vitamin B12 were effective in ameliorating the neurotoxic effect of PPA. The three groups treated with $\omega-3$, vitamin B12, or a combination of both were not significantly different from the control group but were significantly different from the PPA-treated groups. Figure 2 illustrates the overexpression of cPLA2 and the marked attenuation of its expression in PPA-intoxicated and B12- and $\omega$-3-treated groups, respectively.

Table 2 presents the area under the curve (AUC), together with cutoff values, specificity and sensitivity, for CPLA2 and total phospholipids in PPA-intoxicated rats and those treated with either B12 or $\omega-3$ independently or in combination.

\section{Discussion}

In the present study, the significant increase in cPLA2, together with the significant decrease in brain phospholipids, 
Table 1 Mean \pm S.D. of the measured parameters in the five groups studied

\begin{tabular}{|c|c|c|c|c|c|c|c|}
\hline Parameter & Group & $\mathrm{N}$ & Min. & Max. & Mean \pm S.D. & $p$ value $^{\mathbf{a}}$ & $p$ value $^{\mathbf{b}}$ \\
\hline \multirow[t]{5}{*}{ PLA2 } & Control & 7 & 1160.00 & 2091.90 & $1441.00 \pm 317.85$ & & 0.009 \\
\hline & PPA & 7 & 1106.00 & 2290.10 & $1852.28 \pm 393.77$ & 0.087 & \\
\hline & $P P A+B 12$ & 7 & 829.48 & 1357.13 & $1153.71 \pm 214.77$ & 0.318 & \\
\hline & $P P A+\omega 3$ & 7 & 923.35 & 1872.70 & $1364.70 \pm 307.00$ & 0.978 & \\
\hline & $\mathrm{PPA}+\omega-3+\mathrm{B} 12$ & 7 & 853.91 & 1778.19 & $1417.81 \pm 389.19$ & 1.000 & \\
\hline \multirow[t]{5}{*}{ Phospholipid } & Control & 7 & 13.08 & 21.62 & $16.76 \pm 2.80$ & & 0.100 \\
\hline & PPA & 7 & 7.35 & 16.13 & $12.21 \pm 3.17$ & 0.027 & \\
\hline & $\mathrm{PPA}+\mathrm{B} 12$ & 7 & 10.72 & 20.18 & $13.77 \pm 3.33$ & 0.211 & \\
\hline & PPA+ $\omega-3$ & 7 & 10.48 & 21.41 & $14.07 \pm 3.48$ & 0.290 & \\
\hline & $\mathrm{PPA}+\omega-3+\mathrm{B} 12$ & 7 & 12.68 & 16.79 & $14.82 \pm 1.96$ & 0.566 & \\
\hline
\end{tabular}

${ }^{a} p$ value between each group and the control group

${ }^{\mathrm{b}} p$ value among all groups

can be easily related to the neurotoxic effect of PPA. The increase in the expression of PLA2 in the PPA-treated group was clearly detected from the WB when compared with the control (Fig. 2). The ameliorating effects of B12, $\omega-3$, and a mixture of both were also clear in the same blot, which confirms the ELISA data obtained and presented in Table 1 and Fig. 1. This result could be related to the effect of vitamin $B 12$ on $\Delta 5$ and $\Delta 6$ desaturases, two important enzymes critical for the synthesis of PUFA such as EPA, DHA, and AA, in a multistage process of successive catalytic reactions by elongase and desaturase enzymes. Wadhwani et al. [26] proved the effectiveness of vitamin B12 micronutrient supplementation on fatty acid desaturases. Compared with controls, vitamin B12 deficiency during pregnancy is known to reduce $\Delta 6$ desaturase activity and mRNA levels of $\Delta 5$. Interestingly, the mRNA level of $\Delta 5$ desaturase reverts back to normal levels as a result of omega-3 fatty acid supplementation.

The detection of cPLA2 with an average molecular weight of $108 \mathrm{KDa}$ using the WB technique is consistent with the work of Hirashima et al. [27] in which a $110 \mathrm{kDa}$ PLA2 was purified and characterized from bovine brain cytosol. Activation of cPLA2 is usually accompanied by cascade signaling and production of PGE2 as an inflammatory marker. Recognition of the significance of PLA2 in neurodevelopmental inflammatory diseases has made it a very attractive target for drugs. In the present study, B12, $\omega-3$, and a combination of both demonstrate potency in reducing the inflammatory effects of PPA through the attenuation of the increased level of CPLA2 and the decreased concentration of rat brain phospholipids, two neurotoxic features of PPA in the rodent model of autism. This finding is supported by the recent work of Lia et al. [28] in which PLA2 and COX-2 were among the proteins that induced a response to NF- $\mathrm{KB}$ activation after subcutaneous injection of aluminum into a mouse as a xenobiotic with immune-stimulating and neurotoxic effects that might be related to the remarkable increase of autism through the routine exposure of infants to aluminum worldwide.

The significant decrease of phospholipids (Table 1) can be related to glutamate excitotoxicity, the neurotoxic mechanism of PPA [24] and to the etiological mechanism repeatedly reported in autistic patients [29]. Based on the

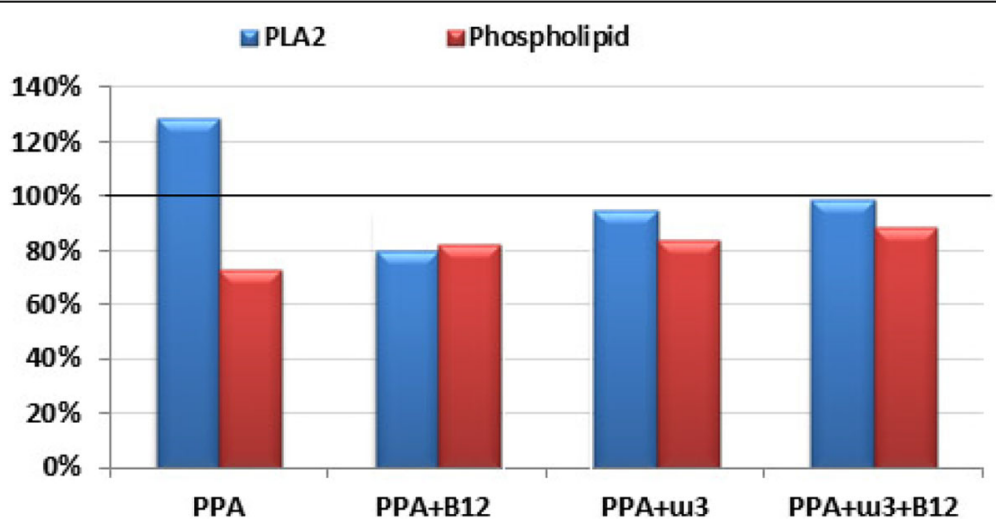

Fig. 1 Percentage change of CPLA2 and phospholipids levels in the four studied groups relative to the control group 


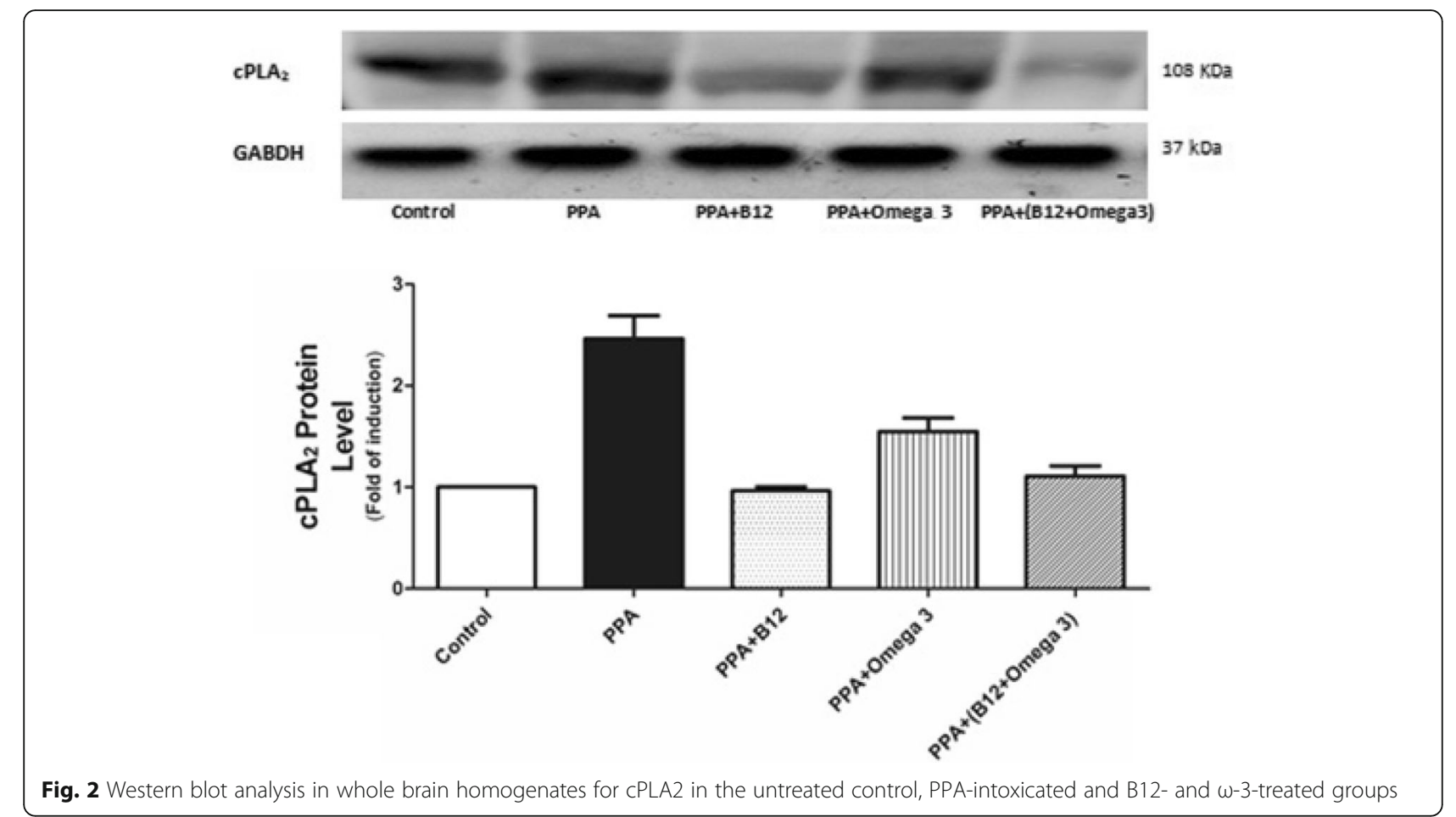

most recent finding of Schneider et al. [30], the plasticity related gene 1 (PRG-1) plays a critical role in decreasing presynaptic glutamate release through the interaction with phospholipids. This result was confirmed when deletion of the PRG-1 gene increased glutamate release and increased neuronal glutamate over-excitation. Based on this finding, the significant decrease of phospholipids in the PPA-treated group of this study can be explained by the glutamate excitotoxicity recently reported in juvenile rats with PPA administered orally [24].

Due to the enhanced neurogenesis during brain development early in life, sufficient dietary sources of $\omega-3$ PUFA are needed for maintenance of neuronal functions [31, 32]. This supplement is mostly needed for the synaptic membranes which contain high levels of phospholipids
[33]. Findings from animal and clinical studies support the role of $\omega-3$ PUFA as essential nutrients for maintaining normal brain function [34].

Based on the fact that autistic patients and the rodent model of autism demonstrate differences in plasma $\omega-3 /$ $\omega-6$ ratios [35-39], $\omega-3$ was suggested as a treatment for autism. The ameliorating effect of $\omega-3$ reported in the present study, shown as a decrease in cPLA2 protein (using ELISA and WB) and the significant increase in the depleted phospholipids, may support the recent interest in the use of this fatty acid as a therapeutic strategy to treat autism. Supplementation with $\omega-3$ for 12 weeks for autistic children ranging from 7 to 18 years old produces significant improvements in all subscales, including blood fatty acid profiles. Unfortunately, once a child already

Table 2 ROC-Curve of all parameters in all groups

\begin{tabular}{|c|c|c|c|c|c|c|}
\hline Parameter & Group & AUC & Cut-off value & Sensitivity \% & Specificity\% & $p$ value \\
\hline \multirow[t]{4}{*}{ CPLA2 } & PPA & 0.755 & 1527.550 & $85.7 \%$ & $85.7 \%$ & 0.110 \\
\hline & $\mathrm{PPA}+\mathrm{B} 12$ & 0.776 & 1398.601 & $100.0 \%$ & $57.1 \%$ & 0.085 \\
\hline & $P P A+\omega-3$ & 0.571 & 1403.250 & $71.4 \%$ & $57.1 \%$ & 0.655 \\
\hline & $\mathrm{PPA}+\omega-3+\mathrm{B} 12$ & 0.510 & 1068.040 & $28.6 \%$ & $100.0 \%$ & 0.949 \\
\hline \multirow[t]{4}{*}{ Phospholipid } & PPA & 0.878 & 15.563 & $85.7 \%$ & $71.4 \%$ & 0.018 \\
\hline & $\mathrm{PPA}+\mathrm{B} 12$ & 0.816 & 12.944 & $71.4 \%$ & $100.0 \%$ & 0.048 \\
\hline & $\mathrm{PPA}+\omega-3$ & 0.816 & 14.345 & $85.7 \%$ & $85.7 \%$ & 0.048 \\
\hline & $\mathrm{PPA}+\omega-3+\mathrm{B} 12$ & 0.735 & 16.536 & $85.7 \%$ & $57.1 \%$ & 0.142 \\
\hline
\end{tabular}


shows the autistic phenotype, $\omega-3$ is unlikely to completely correct the deficits, because there are many other factors involved as etiological mechanisms of this disorder. However, many studies do not exclude the possibility that a subgroup of autistic patients may respond to $\omega-3$ treatment [40]. The remarkable improvement in CPLA2 and phospholipid levels in groups treated with $\omega-3$, vitamin B12, and $\omega-3$ + vitamin B12 is consistent with a recent study by Rathod et al. [41] in which the combined supplementation of vitamin B12 and $\omega-3$ fatty acids led to higher $\omega-3$ and nerve growth factor (NGF) levels in the hippocampus, higher brain derived neurotrophic factor (BDNF) levels in the cortex and hippocampus, together with improvement of cognitive performance. Omega- 3 as a precursor for active mediators can regulate various brain functions such as neurotransmission, inflammation, immune reaction and neuronal survival [42-46].

The present study is consistent with the recent work of Qasem et al. [47], which reported a remarkable reduction in phospholipids and an increase in CPLA2 protein levels as etiological mechanisms of autism; these findings might support the use of PPA postnatal exposure to induce persistent autistic features, similar to the mechanism that causes autism. Receiver operating characteristic analysis demonstrated that while cPLA2 can be used as a marker of PPA neurotoxicity (AUC of $0.755,85.7 \%$ specificity, and 85.7\% sensitivity), phospholipids can be used to predict both PPA neurotoxicity and the therapeutic potency of B12, $\omega-3$ and their mixture (AUCs of $0.878,0.816,0.816$, and 0.735 respectively) with satisfactory specificity and sensitivity [48].

\section{Conclusion}

In the present study, it is likely that vitamin B12 and $\omega-3$ fatty acids may act independently or synergistically to ameliorate the neurotoxic effects of impairment of cPLA2 and phospholipids induced in rat pups by orally administered PPA.

\section{Abbreviations}

AA: Arachidonic acid; CPLA2: Cytosolic phospholipase A2; DG: Diacylglycerol; ELISA: Enzyme-linked immunosorbent assay; IP3: Inositol trisphosphates: PC: Phosphatidylcholine; PE: Phosphatidylethanolamine;

PEpl: Phosphatidylethanolamine plasmalogen; PGs: Prostaglandins; PKC: Protein kinase C; PLA2: Phospholipase A2; PLC: Phospholipase C; PPA: Propionic acid; PS: Phosphatidylserine; WB: Western blot

\section{Acknowledgments}

This research project was supported by a grant from the Research Center of the Center for Female Scientific and Medical Colleges at King Saud University, Riyadh, Saudi Arabia.

\section{Funding}

Research Center of the Center for Female Scientific and Medical Colleges at King Saud University, Riyadh, Saudi Arabia.

Availability of data and materials

All data explored during this study are included in the article.

\section{Authors' contributions}

HA, Suggested the topic and codrafted the manuscript; RS, Codrafted the manuscript; MA, Acquisition of data; OM, Acquisition of data; AA, Codrafted the manuscript; MA, Performed the statistical analysis; MA, Acquisition of data; $I H$, Acquisition of data; $A E$, Suggested the topic and drafted the manuscript. All authors read and approved the final manuscript.

\section{Ethics approval}

All animal experiments were conducted with the approval of King Saud University.

\section{Consent for publication}

Not applicable.

\section{Competing interests}

The authors declare that they have no competing interests.

\section{Publisher's Note}

Springer Nature remains neutral with regard to jurisdictional claims in published maps and institutional affiliations.

\section{Author details}

${ }^{1}$ Department of Food Science and Nutrition, College of Food and Agriculture Sciences, King Saud University, P.O. Box 22452, Riyadh, Saudi Arabia.

${ }^{2}$ Biochemistry Department, College of Science, King Saud University, Riyadh 11451, Kingdom of Saudi Arabia. ${ }^{3}$ Central Laboratory, Center for Female Scientific and Medical Colleges, King Saud University, Riyadh, Saudi Arabia. ${ }^{4}$ Pharmacology and Toxicology Department, College of Pharmacy, Riyadh, Saudi Arabia.

Received: 1 June 2018 Accepted: 13 August 2018

Published online: 31 August 2018

\section{References}

1. El-Ansary AK, Ben Bacha A, Kotb M. Etiology of autistic features: the persisting neurotoxic effects of propionic acid. J Neuroinflammation. 2012;9:74.

2. Khalil SR, Abd-Elhakim YM, Selim ME, Al-Ayadhi LY. Apitoxin protects rat pups brain from propionic acid-induced oxidative stress: the expression pattern of $\mathrm{BCl}-2$ and Caspase-3 apoptotic genes. Neurotoxicology. 2015;49:121-31.

3. Daghestani MH, Selim ME, Abd-Elhakim YM, Said EN, El-Hameed NEA, Khalil SR, El-Tawil OS. The role of apitoxin in alleviating propionic acid-induced neurobehavioral impairments in rat pups: the expression pattern of Reelin gene. Biomed Pharmacother. 2017;93:48-56H.Y.

4. Farooqui AA, Liss L, Horrocks LA. Neurochemical aspects of alzheimers-disease - involvement of membrane phospholipids. Metab Brain Dis. 1988;3:19-35.

5. Brown CM, Austin DW. Autistic disorder and phospholipids: a review. Prostaglandins Leukot Essent Fatty Acids. 2011;84(1-2):25-30.

6. Kim BX, Huang AAS. Phosphatidylserine in the brain: metabolism and function. Prog Lipid Res. 2014;56:1-18.

7. Sun GY, Horrocks LA. The fatty acid and aldehyde composition of the major phospholipids of mouse brain. Lipids. 1968;3:79-83.

8. Sun GY, Horrocks LA. The acyl and alk-1-enyl groups of the major phosphoglycerides from ox brain myelin and mouse brain microsomal, mitochondrial and myelin fractions. Lipids. 1970;5:1006-12.

9. Hayaishi O. Molecular mechanisms of sleep-wake regulation: roles of prostaglandin D2 and E2. Fedn Am Socs Exp Biol J. 1991;5:2575-81.

10. Matsumura $\mathrm{K}, \mathrm{Cao} C$, Yu W, Watanabe Y. Prostaglandin system in the brain: sites of biosynthesis and sites of action under normal and hyperthermic states. Prog Brain Res. 1998;115:275-95.

11. McCann JC, Ames BN. Is docosahexaenoic acid, an n-3 long-chain polyunsaturated fatty acid, required for development of normal brain function? An overview of evidence from cognitive and behavioral tests in humans and animals. Am J Clin Nutr. 2005;82:281-95.

12. Kidd PM. Omega-3 DHA and EPA for cognition, behavior, and mood: clinical findings and structural-functional synergies with cell membrane phospholipids. Altern Med Rev. 2007;12(3):207-27.

13. Breckenridge WC, Gombos G, Morgan IG. The lipid composition of adult rat brain synaptosomal plasma membranes. Biochim Biophys Acta. 1972;266: 695-707. 6. Neuringer M, Connor WE, Lin DS, et al 
14. Shimizu T, Wolfe L-S. Arachidonic acid cascade and signal transduction. J Neurochem. 1990;55:1-15.

15. Murakami M, Kudo I. Phospholipase A2. J Biochem. 2002;131(3):285-92.

16. Green JT, Orr SK, Bazinet RP. The emerging role of group VI calciumindependent phospholipase A2 in releasing docosahexaenoic acid from brain phospholipids. J Lipid Res. 2008;49(5):939-44.

17. Massicotte G. Modification of glutamate receptors by phospholipase A2: its role in adaptive neural plasticity. Cell Mol Life Sci. 2000:57(11):1542-50.

18. Saluja I, Song D, O'Regan MH, Phillis JW. Role of phospholipase A2 in the release of free fatty acids during ischemia-reperfusion in the rat cerebral cortex. Neurosci Lett. 1997;233(2-3):97-100.

19. Kang X, Qiu J, Li Q, Bell KA, Du Y, Jung DW, Lee JY, Hao J, Jiang J. Cyclooxygenase-2 contributes to oxidopamine-mediated neuronal inflammation and injury via the prostaglandin E2 receptor EP2 subtype. Sci Rep. 2017;7(1):9459.

20. Bosetti F, Weerasinghe GR. The expression of brain cyclooxygenase- 2 is down-regulated in the cytosolic phospholipase A2 knockout mouse. J Neurochem. 2003;87:1471-7.

21. Rosenberger TA, Villacreses NE, Contreras MA, Bonventre JV, Rapoport SI. Brain lipid metabolism in the cPLA2 knockout mouse. J Lipid Res. 2003:44(1):109-17.

22. Bosetti F. Arachidonic acid metabolism in brain physiology and pathology: lessons from genetically altered mouse models. J Neurochem. 2007;102:577-86.

23. El-Ansary A, Al-Salem HS, Asma A, Al-Dbass A. Glutamate excitotoxicity induced by orally administered propionic acid, a short chain fatty acid can be ameliorated by bee pollen. Lipids Health Dis. 2017;16(1):96.

24. Ma H, Shieh K. Western blotting method. J Am Sci. 2006;2:23-7.

25. Wyatt I, Farnworth M, Gyte AJ, Lock EA. L-2-chloropropionic acid metabolism and disposition in male rats: relevance to cerebellar injury. Arch Toxicol. 1997;71:668

26. Hirashima Y, Farooqui AA, Mills JS, Horrocks LA. Identification and Puri- fication of calcium-independent phospholipase A2 from bovine brain cytosol. J Neurochem. 1992;59:708e714.

27. Wadhwani NS, Manglekar RR, Dangat KD, Kulkarni AV, Joshi SR. Effect of maternal micronutrients (folic acid, vitamin B12) and omega 3 fatty acids on liver fattyacid desaturases and transport proteins in Wistar rats. Prostaglandins Leukot Essent Fatty Acids. 2012;86(1-2):21-7.

28. Lia D, Tomljenovica L, Lia Y, Shaw CA. Subcutaneous injections of aluminum at vaccine adjuvant levels activate innate immune genes in mouse brain that are homologous with biomarkers of autism. J Inorg Biochem. 2017;177:39-54.

29. El-Ansary A, Al-Ayadhi L. GABAergic/glutamatergic imbalance relative to excessive neuroinflammation in autism spectrum disorders. J Neuroinflammation. 2014;11:189.

30. Schneider P, Petzold S, Sommer A, Nitsch R, Schwegler H, Vogt J. Roskoden altered synaptic phospholipid signaling in PRG1 deficient mice inducesexploratory behavior and motor hyperactivity resembling psychiatric disorders. Behav Brain Res. 2018;336:1-7.

31. Al-Amin MM, Rahman MM, Khan FR, Zaman F, Mahmud Reza H. Astaxanthin improves behavioral disorder and oxidative stress in prenatal valproic acidinduced mice model of autism. Behav Brain Res. 2015;286:112-21.

32. Hashimoto M. N-3 fatty acids and the maintenance of neuronal functions. Folia Pharmacol Jpn. 2018;151(1):27-33.

33. Tanaka K, Farooqui AA, Yi O w. Effects of docosahexaenoic acid on neurotransmission. Biomol Ther. 2012;20(2):152-7.

34. Weiser MJ, Mucha B, Denheyer H, Atkinson D, Schanz N, Vassiliou E, Benno $\mathrm{RH}$. Dietary docosahexaenoic acid alleviates autistic like behaviors resulting from maternal immune activation in mice. Prostaglandins Leukot Essent Fatty Acids. 2016;106:27-37.

35. Al-Farsi YM, Waly Ml, Deth RC, Al-Sharbati MM, Al-Shafaee M, Al-Farsi O, Al-Khaduri MM, Al-Adawi S, Hodgson NW, Gupta I, Ouhtit A. Impact of nutrition on serum levels of docosahexaenoic acid among Omani children with autism. Nutrition. 2013;29:1142-6.

36. El-Ansary AK, Bacha AG, Al-Ayahdi LY. Impaired plasma phospholipids and relative amounts of essential polyunsaturated fatty acids in autistic patients from Saudi Arabia. Lipids Health Dis. 2011;10:63.

37. Mostafa GA, . Al-Ayadhi LY, Reduced levels of plasma polyunsaturated fatty acids and serum carnitine in autistic children: relation to gastrointestinal manifestations, Behav Brain Funct 2015; 11:4

38. Sliwinski S, Croonenberghs J, Christophe A, Deboutte D, Maes M. Polyunsaturated fatty acids: do they have a role in the pathophysiology of autism? Neuro Endocrinol Lett. 2006;27:465-71.
39. Vancassel S, Durand G, Barthelemy C, Lejeune B, Martineau J, Guilloteau D, Andres C, Chalon S. Plasma fatty acid levels in autistic children. Prostaglandins Leukot Ess Fat Acids. 2001;65:1-7.

40. Posar A, Visconti P. Omega-3 supplementation in autism spectrum disorders: a still open question? J Pediatr Neurosci. 2016;11:225-7.

41. Rathod RS, Khaire AA, Kale AA, R Joshi SR. Beneficial effects of omega-3 fatty acids and vitamin B12 supplementation on brain docosahexaenoic acid, brain derived neurotrophic factor, and cognitive performa. Biofactors. 2016:41(4):261-72.

42. Sun GY, Simonyi A, Fritsche KL, Chuang DY, Hannink M, Gu Z, Greenlief CM, Yao JK, Lee JC, Beversdorf DQ. Docosahexaenoic acid (DHA): an essential nutrient and a nutraceutical for brain health and diseases. Prostaglandins Leukot Essent Fatty Acids. 2017;16:30213-7.

43. Das UN. Autism as a disorder of deficiency of brain-derived neurotrophic factor and altered metabolism of polyunsaturated fatty acids. Nutrition. 2013;29(10):1175-85.

44. Wu A, Ying Z, Gomez-Pinilla F. The salutary effects of DHA dietary supplementation on cognition, neuroplasticity, and membrane homeostasis after brain trauma. J Neurotrauma. 2011;28:2113e2122

45. Wu A, Ying Z, Gomez-Pinilla F. Dietary omega-3 fatty acids normalize BDNF levels, reduce oxidative damage, and counteract learning disability after traumatic brain injury in rats. J Neurotrauma. 2004;21:1457e1467.

46. SanGiovanni JP, Chew EY. The role of omega-3 long-chain polyunsaturated fatty acids in health and disease of the retina. Prog Retin Eye Res. 2005;24:87e138.

47. Qasem H, Al-Ayadhi L, Al Dera H, El-Ansary A. Increase of cytosolic phospholipase A2 as hydrolytic enzyme of phospholipids and autismcognitive, social and sensory dysfunction severity. Lipids Health Dis. 2017;16(1):117.

48. Pinches M, Betts C, Bickerton S, Burdett L, Thomas H, Derbyshire N, Jones HB, Moores M. Evaluation of novel renal biomarkers with a cisplatin model of kidney injury: gender and dosage differences. Toxicol Pathol. 2012;40(3):522-33.

\section{Ready to submit your research? Choose BMC and benefit from:}

- fast, convenient online submission

- thorough peer review by experienced researchers in your field

- rapid publication on acceptance

- support for research data, including large and complex data types

- gold Open Access which fosters wider collaboration and increased citations

- maximum visibility for your research: over $100 \mathrm{M}$ website views per year

At BMC, research is always in progress.

Learn more biomedcentral.com/submissions 